\title{
Rosuvastatin Ameliorates Inflammation, Renal Fat Accumulation, and Kidney Injury in Transgenic Spontaneously Hypertensive Rats Expressing Human C-Reactive Protein
}

\author{
J. ŠILHAVÝ ${ }^{1}$, V. ZÍDEK ${ }^{1}$, V. LANDA ${ }^{1}$, M. ŠIMÁKOVÁ ${ }^{1}$, P. MLEJNEK ${ }^{1}$, O. OLIYARNYK ${ }^{2}$, \\ H. MALÍNSKÁ ${ }^{2}$, L. KAZDOVÁ ${ }^{2}$, M. MANCINI ${ }^{3}$, M. PRAVENEC ${ }^{1}$ \\ ${ }^{1}$ Institute of Physiology, Czech Academy of Sciences, Prague, Czech Republic, ${ }^{2}$ Center of \\ Experimental Medicine, Institute for Clinical and Experimental Medicine, Prague, Czech Republic, \\ ${ }^{3}$ Department of Radiology, Oncology and Pathology, Sapienza Università di Roma, Rome, Italy
}

Received April 4, 2014

Accepted November 4, 2014

On-line December 22, 2014

\section{Summary}

Recently, we derived "humanized" spontaneously hypertensive rats (SHR-CRP) in which transgenic expression of human CRP induces inflammation, oxidative stress, several features of metabolic syndrome and target organ injury. In addition, we found that rosuvastatin treatment of SHR-CRP transgenic rats can protect against pro-inflammatory effects of human CRP and also reduce cardiac inflammation and oxidative damage. In the current study, we tested the effects of rosuvastatin $(5 \mathrm{mg} / \mathrm{kg})$ on kidney injury in SHR-CRP males versus untreated SHR-CRP and SHR controls. All rats were fed a high sucrose diet. In SHR-CRP transgenic rats, treatment with rosuvastatin for 10 weeks, compared to untreated transgenic rats and SHR controls, was associated with significantly reduced systemic inflammation which was accompanied with activation of antioxidative enzymes in the kidney, lower renal fat accumulation, and with amelioration of histopathological changes in the kidney. These findings provide evidence that, in the presence of high CRP levels, rosuvastatin exhibits significant anti-inflammatory, anti-oxidative, and renoprotective effects.

\section{Key words}

Rosuvastatin - Kidney damage - CRP - Transgenic • Spontaneously hypertensive rat

\section{Corresponding author}

M. Pravenec, Institute of Physiology, Czech Academy of Sciences, Vídeňská 1083, 14220 Prague 4, Czech Republic. Fax: (420) 241062488. E-mail: pravenec@biomed.cas.cz

\section{Introduction}

C-reactive protein (CRP) is a widely used biomarker of acute systemic inflammation. In addition, CRP levels are used as a predictor for overall mortality in patients with chronic kidney disease or end stage renal disease (Zhang et al. 2013). However, the extent to which CRP itself promotes inflammation and contributes to the pathogenesis of kidney disease is highly controversial (Scirica et al. 2006). Recently, we derived "humanized" transgenic strain of spontaneously hypertensive rat (SHR-CRP transgenic) in which expression of human CRP in the liver is associated with increased levels of circulating human CRP, systemic inflammation, metabolic and hemodynamic disturbances, and target organ injury, including increases in albuminuria and histopathological changes such as fibrosis and inflammatory cellular infiltrates in the interstitium of the kidney (Pravenec et al. 2011). These findings suggest that expression of transgenic CRP in the SHR and associated inflammation might be causally related to kidney injury. Statins are recommended for treatment of patients with stage 1-3 of chronic kidney disease and with increased levels of LDL cholesterol because of possible role of dyslipidemia in the pathogenesis of kidney disease (Qaseem et al. 2013). However, results of several metaanalyses showed that renoprotective role of statins in patients with chronic kidney disease is controversial, 
depending on stage of the disease, presence of diabetes, specific statin used, ethnicity, etc. (Hou et al. 2013, Nicolic et al. 2013, Palmer et al. 2014). In addition to decreasing LDL cholesterol levels, pleiotropic effects of statins include reduction of inflammation and oxidative stress (Yagi et al. 2012). Recently, we found that rosuvastatin treatment of SHR-CRP transgenic rats decreased circulating levels of inflammatory response markers IL6 and TNF $\alpha$ and reduced cardiac inflammation and oxidative damage (Šilhavý et al. 2014). In the current study in SHR-CRP transgenic rats, we investigated whether rosuvastatin could protect kidneys against inflammation, oxidative stress, ectopic fat accumulation, and tissue injury.

\section{Methods}

Animals

Transgenic SHR (hereafter referred to as SHR-CRP transgenic) were derived by microinjections of SHR ova with a previously described construct containing the cDNA for human CRP under control of the apoE promoter (Koike et al. 2009) with the objective of driving expression of the CRP transgene in liver where CRP is normally produced (Pravenec et al. 2011). To investigate effects of rosuvastatin on kidney injury associated with human CRP, we randomized 12 month old male SHR-CRP transgenic to groups with or without rosuvastatin treatment and also included age matched untreated control group of male non-transgenic SHR. SHR-CRP transgenic rats were treated with rosuvastatin $(5 \mathrm{mg} / \mathrm{kg} /$ day) in the drinking water for 10 weeks. In each group, we studied 8 animals. All rats were fed standard rat chow for the first 12 months and then switched to a high sucrose diet ( $60 \%$ sucrose) to increase risk of developing metabolic disturbances during the 10 week study period. The rats were housed in an air-conditioned animal facility and allowed free access to sucrose diet and water. All experiments were performed in agreement with the Animal Protection Law of the Czech Republic and were approved by the Ethics Committee of the Institute of Physiology, Academy of Sciences of the Czech Republic, Prague.

\section{Tissue triglyceride measurements}

The kidney tissue was powdered under liquid $\mathrm{N}_{2}$ and extracted for $16 \mathrm{~h}$ in chloroform:methanol, after which $2 \% \mathrm{KH}_{2} \mathrm{PO}_{4}$ was added and the solution was centrifuged. The organic phase was removed and evaporated under $\mathrm{N}_{2}$. The resulting pellet was dissolved in isopropyl alcohol, and triglyceride content was determined by enzymatic assay (Erba-Lachema, Brno, Czech Republic).

Urine collection, microalbuminuria, creatinine, urine cGMP, and glomerular filtration rate

Rats were placed into metabolic cages for $16 \mathrm{~h}$ to obtain urine samples for analysis of urinary excretion of albumin. The level of albumin in urine was analyzed by HPLC method with UV-VIS detection according to Contois et al. (2006). Urine albumin was adjusted for creatinine concentration $(\mathrm{mg} / \mathrm{g}$ creatinine). Urine and serum creatinine was measured by Jaffe rate assay (Erba-Lachema, Brno, Czech Republic). Urine cGMP was determined by immunoassay (Immunotech, France). Glomerular filtration rate (GFR) was calculated according to the formula $\mathrm{GFR}=$ Urine creatinine concentration $\mathrm{x}$ Urine flow/Plasma creatinine concentrations.

\section{Parameters of oxidative stress}

The activity of antioxidative enzymes and concentrations of lipoperoxidation products were measured as previously described (Malínská et al. 2010). The activity of superoxide dismutase (SOD) was analyzed using the reaction of blocking nitrotetrazolium blue reduction and nitroformazan formation. Catalase (CAT) activity measurement was based on the ability of $\mathrm{H}_{2} \mathrm{O}_{2}$ to produce with ammonium molybdate a color complex detected spectrophotometrically. The activity of seleno-dependent glutathione peroxidase (GSH-Px) was monitored by oxidation of gluthathione by Ellman reagent ( $0.01 \mathrm{M}$ solution of 5,5'-dythiobis-2 nitrobenzoic acid). The level of reduced glutathione (GSH) was determined in the reaction of SH-groups using Ellman reagent. Glutathione reductase (GR) activity was measured by the decrease of absorbance at $340 \mathrm{~nm}$ using a millimolar extinction coefficient of $6220 \mathrm{M}^{-1} \mathrm{~cm}^{-1}$ for NADPH (using Sigma assay kit). The levels of conjugated dienes (CD) were analyzed by extraction in the media (heptane:isopropanol $=2: 1$ ) and measured spectrophotometrically in the heptane layer. The levels of thiobarbituric acid reactive substances (TBARS) were determined by the reaction with thiobarbituric acid.

\section{Histolgy}

Kidneys $(\mathrm{N}=5)$ from each group (SHR-CRP, SHR-CRP + rosuvastatin and SHR wild type) were cut 
along long axis and processed for paraffin embedding. Multiple $4 \mu \mathrm{m}$ thick section were cut and stained with Hematoxylin-Eosin, PAS and Azan-Mallory trichrome stain for observation under light microscopy. Slides were observed and picture acquired with a digitalized camera by an experienced pathologist blinded by the groups.

\section{Statistical analysis}

All data are expressed as means \pm SEM. Differences between experimental groups were analyzed by one away ANOVA with adjustments for multiple comparisons by Holm Sidak testing. Statistical significance was defined as $\mathrm{P}<0.05$.

\section{Results}

Effects of rosuvastatin on body weight, relative heart and kidney weight, microalbuminuria, urine cGMP concentration, glomerular filtration rate, and renal lipids

Table 1 shows body and organ weights in SHRCRP rats treated with rosuvastatin or placebo and in SHR controls. As can be seen, there were no significant effects of treatment of relative heart and kidney weight. Treatment of SHR-CRP transgenic rats with rosuvastatin was associated with significantly reduced microalbuminuria when compared to untreated SHR-CRP rats, however, both untreated SHR-CRP and rosuvastatin treated SHR-CRP rats exhibited significantly higher microalbuminuria when compared to nontransgenic SHR controls (Fig. 1A). In addition, we have found that levels of the major nitric oxide second messenger cyclic GMP (cGMP) are significantly increased in urine collected from SHR-CRP transgenic rats treated with rosuvastatin when compared to untreated SHR-CRP rats and are similar to those found in nontransgenic SHR (Fig. 1B). There were no significant differences in GFR among the 3 experimental groups (data not shown). Furthermore, SHR-CRP transgenic rats treated with rosuvastatin had significantly reduced ectopic fat accumulation in their kidneys when compared to untreated SHR-CRP and SHR controls (Fig. 1C).

A. Microalbuminuria

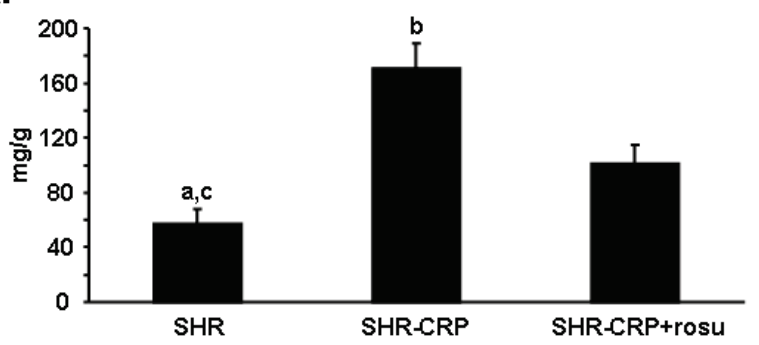

B.

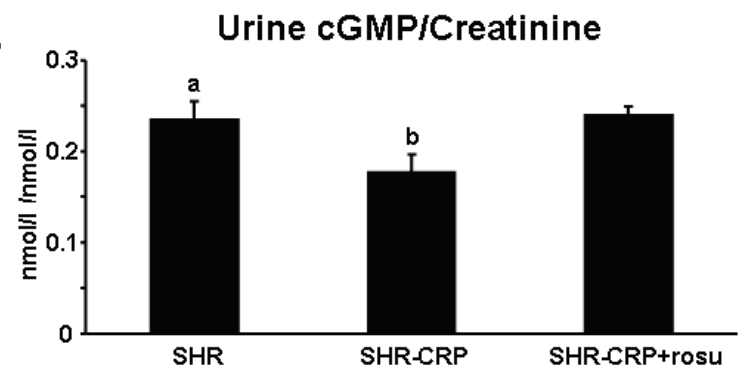

C.

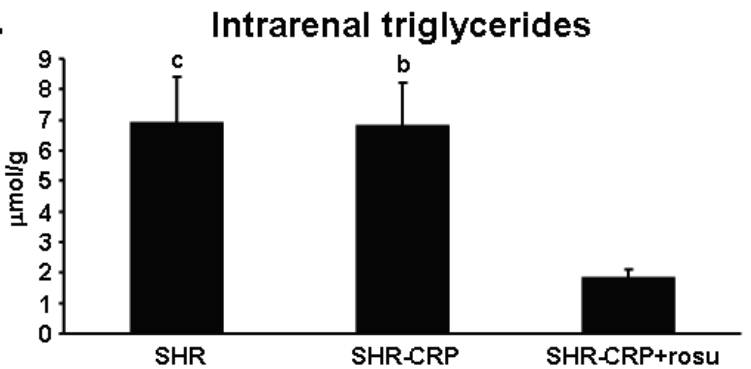

Fig. 1. Levels of microalbuminuria, urine cGMP (mg)/ creatinine (g) ratio, and kidney fat content in SHR nontransgenic and SHR-CRP transgenic untreated rats and SHR-CRP transgenic rats treated with rosuvastatin. Significant differences between the groups are indicated by symbols and were determined by a one-way ANOVA and post-hoc Holm-Sidak test: ${ }^{a}$ denotes $\mathrm{P}<0.05$ SHR versus SHR-CRP, b denotes SHR-CRP versus SHRCRP+rosu, ' denotes SHR versus SHR-CRP+rosu.

Table 1. Body and organ weights in SHR control rats treated with placebo versus SHR-CRP transgenic rats treated with placebo or with rosuvastatin.

\begin{tabular}{lccc}
\hline Trait/Strain & SHR & SHR-CRP & SHR-CRP + rosuvastatin \\
\hline Body weight $(g)$ & $470 \pm 10$ & $443 \pm 7$ & $420 \pm 8^{\mathrm{c}}$ \\
Heart weight $(\mathrm{g} / 100 \mathrm{~g} B \mathrm{~W})$ & $0.35 \pm 0.01^{\mathrm{a}}$ & $0.39 \pm 0.01$ & $0.40 \pm 0.01^{\mathrm{c}}$ \\
Kidney weight $(\mathrm{g} / \mathrm{l} 00 \mathrm{~g} \mathrm{BW})$ & $0.35 \pm 0.01$ & $0.38 \pm 0.01$ & $0.37 \pm 0.01$ \\
\hline
\end{tabular}

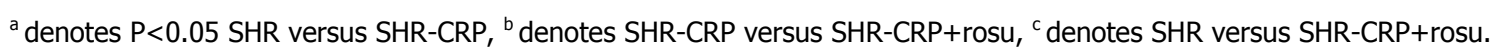


Table 2. Parameters of oxidative stress in renal cortex in SHR control rats treated with placebo versus SHR-CRP transgenic rats treated with placebo or with rosuvastatin.

\begin{tabular}{lccc}
\hline Trait/Strain & SHR & SHR-CRP & SHR-CRP + rosuvastatin \\
\hline$S O D(U / m g)$ & $0.040 \pm 0.003$ & $0.034 \pm 0.003^{\mathrm{b}}$ & $0.056 \pm 0.005^{\mathrm{c}}$ \\
$G S H-P x(\mu \mathrm{M} \mathrm{GSH} / \mathrm{min} / \mathrm{mg})$ & $127 \pm 9^{\mathrm{a}}$ & $96 \pm 6^{\mathrm{b}}$ & $131 \pm 10$ \\
$G R(\mu M \mathrm{NADPH} / \mathrm{min} / \mathrm{mg})$ & $58 \pm 6$ & $68 \pm 4$ & $54 \pm 5$ \\
$\mathrm{CAT}\left(\mathrm{mM} \mathrm{H} \mathrm{H}_{2} \mathrm{O}_{2} / \mathrm{min} / \mathrm{mg}\right)$ & $687 \pm 38$ & $643 \pm 41$ & $730 \pm 36$ \\
$G S H(\mathrm{mM} / \mathrm{g})$ & $9.3 \pm 0.8^{\mathrm{a}}$ & $5.9 \pm 0.3^{\mathrm{b}}$ & $9.5 \pm 0.8$ \\
$C D(\mathrm{nM} / \mathrm{mg})$ & $13.1 \pm 1.0$ & $14.0 \pm 0.8$ & $13.8 \pm 1.1$ \\
$T B A R S(\mathrm{nM} / \mathrm{mg})$ & $0.74 \pm 0.06$ & $0.83 \pm 0.06$ & $0.92 \pm 0.09$ \\
\hline
\end{tabular}

${ }^{a}$ denotes $\mathrm{P}<0.05$ SHR versus SHR-CRP, ${ }^{\text {b }}$ denotes SHR-CRP versus SHR-CRP+rosu, ${ }^{c}$ denotes SHR versus SHR-CRP+rosu.

Effects of rosuvastatin on CRP-induced oxidative stress in the kidney

Table 2 shows parameters of oxidative stress in renal cortex in SHR-CRP transgenic rats treated with placebo or rosuvastatin versus SHR placebo controls. The activity of antioxidative enzyme SOD was increased in SHR-CRP rats treated with rosuvastatin when compared to untreated transgenic rats and SHR controls. The activity of GSH-dependent enzyme, GSH-Px, in SHRCRP rosuvastatin treated rats was similar to SHR controls and significantly higher when compared to untreated SHR-CRP rats. The activity of GSH-regenerating enzyme GR and the activity of catalase were similar among the 3 groups. Levels of GSH in SHR-CRP rosuvastatin treated rats were similar to those in SHR controls and were significantly higher than in untreated SHR-CRP rats. There were no significant differences in levels of lipoperoxidation products, conjugated dienes and TBARS (Table 2).

Effects of rousvastatin on amelioration of CRP-induced kidney inflammatory injury

Qualitative assessment of SHR-CRP animals on PAS and trichrome stained sections showed glomerular fibrosis with thickening of tubular basal membrane, focal inflammatory infiltrate and occasional protein casts in tubular spaces, both these features were absent in treated SHR-CRP rats and nontransgenic SHR controls (Fig. 2A). We also observed reduction of glomerular density in SHR-CRP rats compared to treated SHR-CRP transgenic and control SHR (Fig. 2B). In addition, we observed an expansion of mesangial spaces accompanied by increased cellularity which was reverted by rosuvastatin treatment (Fig. 2C).
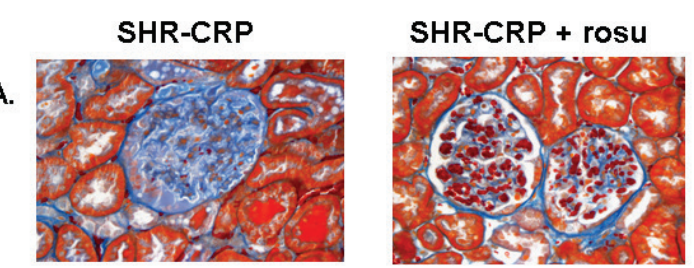

B.
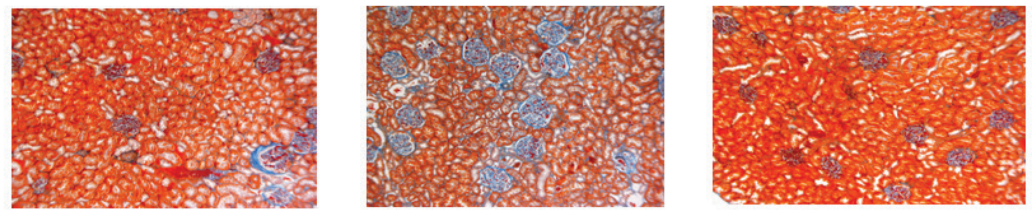

c.

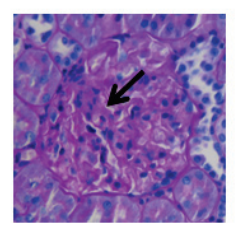

SHR
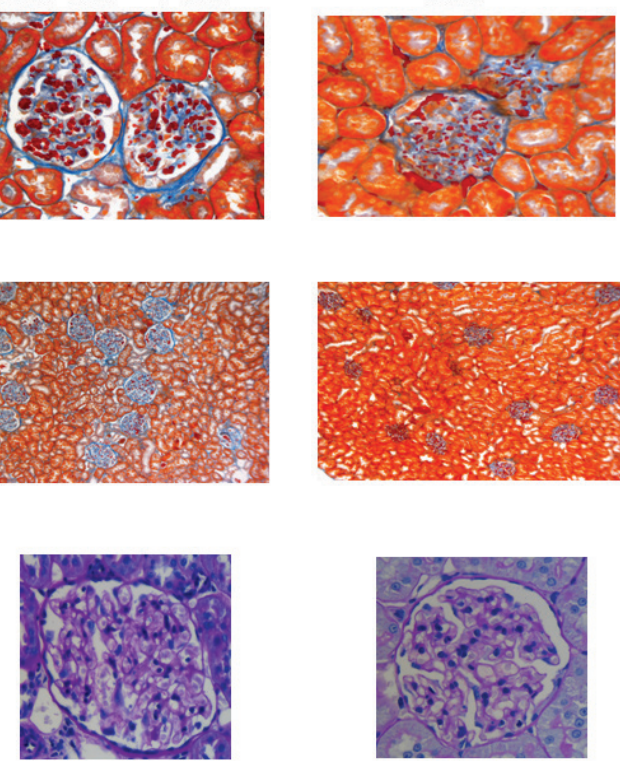

Fig. 2. Histological analysis of kidneys from untreated SHR-CRP transgenic, SHR-CRP transgenic rats treated with rosuvastatin and SHR controls. A. Glomerular fibrosis. Untreated SHR-CRP rats showed extensive glomerular fibrosis and global sclerosis while glomeruli from both rosuvastatin treated group and SHR controls showed no significative change of glomerular morphology. Azan-Mallory stain 40x original magnification. B. Glomerular density. SHRCRP and SHR controls showed decreased glomerular density which was ameliorated in rosuvastatin treated group. Azan-Mallory stain $10 x$ original magnification. C. Mesangial expansion. Glomeruli from SHR-CRP group are characterized by increased cellularity of the glomerular tuft and mesangial axis expansion characterized by both an increase in the number of cells and in deposition of extracellular matrix (arrow). This alterations were ameliorated in rosuvastatin treated animals and SHR controls. PAS stain 40x original magnification. 


\section{Discussion}

The causal role of CRP in the pathogenesis of atherosclerosis, cardiovascular disease, and kidney injury is highly controversial (Rietzschel et al. 2012). There is evidence from animal models of chronic kidney disease suggesting that CRP actively increases inflammation in the kidney (Li et al. 2011, Liu et al. 2011). In our original study, we also observed that transgenic overexpression of human CRP was associated with partial and total sclerosis of glomeruli and with fibrosis and inflammatory cellular infiltrates in the interstitium which provided compelling evidence for causal role of CRP induced inflammation in target organ injury (Pravenec et al. 2011).

Obesity is associated with ectopic fat accumulation and lipotoxicity which may lead to kidney dysfunction (reviewed in Guebre-Egziabher et al. 2013). The mechanisms connecting ectopic fat accumulation with chronic kidney disease remain to be determined. In our previous study (Šilhavý et al. 2014), rosuvastatin treatment in SHR-CRP rats was associated with significant amelioration of insulin resistance in adipose tissue and increase lipolysis. Thus it is possible that resistance of adipose tissue to insulin action in SHR-CRP rats is associated with reduced uptake of fatty acids and glucose, ectopic fat accumulation and lipotoxicity in the kidney. In addition, rosuvastatin treatment was associated with increased levels of the major nitric oxide second messenger cGMP in SHR-CRP transgenic rats. Similar observation was reported in mice expressing human CRP transgene (Grad et al. 2007). It is therefore possible that rosuvastatin ameliorates endothelial dysfuction in the kidney that is exposed to increased levels of human CRP (Mather 2013).

Regarding histologic results, the presence of mixed features of both hypertensive nephroangiosclerosis (Olson 1998) and mesangial expansion typical of diabetic nephropathy in its early stages (Tervaert et al. 2010, Valk et al. 2011) should be noted in the SHR-CRP group. These changes were reverted by rosuvastatin treatment and were extremely mild or absent in the SHR control group. These findings are in agreement with clinical data regarding kidney oxidative stress, microalbuminuria and glomerular filtration rate, suggesting the role of both inflammation and oxidative stress in glomerular injury of hypertensive dysmetabolic patients (Adler 2004, Wolf 2004, Kopp 2013).

In the current studies, we did not measure the effects of rosuvastatin on blood pressure. Because increased blood pressure can promote oxidative stress and inflammation, it should be recognized that the antioxidant and anti-inflammatory effects of rosuvastatin in vivo may also be secondary to the ability of rosuvastatin to limit increases in blood pressure otherwise induced by human CRP. On the other hand, putative blood pressure lowering effects of rosuvastatin might be secondary to its renoprotective effects. Thus it is difficult to distinguish between effects of rosuvastatin on blood pressure and renal protection.

The mechanisms by which rosuvastatin protects kidney against inflammation, oxidative stress, and ectopic fat accumulation are not fully understood. In our recent study (Šilhavý et al. 2014), we determined gene expression profiles in livers isolated from SHR-CRP and SHR rats treated with rosuvastatin or with placebo and identified Neurotrophin signaling and Influenza A as the most significant KEGG pathways that play important role in therapeutic effects of rosuvastatin. These KEGG pathways include genes involved in Toll-like receptor signaling, c-Jun N-terminal MAPK signaling, the extracellular signal-regulated Raf/Mek/Erk signaling, and nuclear factor kappa B signaling as well as some additional genes involved in innate immune and antiviral defensive mechanisms. Thus it is likely that rosuvastatin treatment affects these inflammation regulatory pathways and at the same time decreases kidney fat accumulation by its hypolipidemic effects.

The use of statins in therapy of patients with chronic kidney disease is controversial. Some studies showed that statins might protect renal function while other found no benefits or even increased proteinuria, especially in statins with high cholesterol-lowering efficacy such as rosuvastatin (for review see Kalaitzidis et al. 2011). Results of the current study demonstrate important role of human CRP in the pathogenesis of kidney injury and significant anti-inflammatory, antioxidative, and renoprotective effects of rosuvastatin in the SHR-CRP model. The current study thus raises the possibility that rosuvastatin treatment might be effective especially in humans with renal disease that is accompanied with increased CRP levels.

\section{Conflict of Interest}

There is no conflict of interest.

\section{Acknowledgements}

This work was supported by grant LH11049 to VZ and 
LL1204 (within the ERC CZ program) to MP from the Ministry of Education, Youth and Sports of the Czech Republic, grant NT/14325 to MP from the Ministry of Health of the Czech Republic, grant MH CZ-DRO
("Institute for Clinical and Experimental Medicine IKEM, IN 0002301") to LK, grant 14-36804G from the Czech Science Foundation to MP.

\section{References}

ADLER S: Diabetic nephropathy: linking histology, cell biology, and genetics. Kidney Int 66: 2095-2106, 2004.

CONTOIS JH, HARTIGAN C, RAO LV, SNYDER LM, THOMPSON MJ: Analytical validation of an HPLC assay for urinary albumin. Clin Chim Acta 367: 150-155, 2006.

GRAD E, GOLOMB M, MOR-YOSEF I, KOROUKHOV N, LOTAN C, EDELMAN ER, DANENBERG HD: Transgenic expression of human C-reactive protein suppresses endothelial nitric oxide synthase expression and bioactivity after vascular injury. Am J Physiol Heart Circ Physiol 293: H489-H495, 2007.

GUEBRE-EGZIABHER F, ALIX PM, KOPPE L, PELLETIER CC, KALBACHER E, FOUQUE D, SOULAGE CO: Ectopic lipid accumulation: a potential cause for metabolic disturbances and a contributor to the alteration of kidney function. Biochimie 95: 1971-1979, 2013.

HOU W, LV J, PERKOVIC V, YANG L, ZHAO N, JARDINE MJ, CASS A, ZHANG H, WANG H: Effect of statin therapy on cardiovascular and renal outcomes in patients with chronic kidney disease: a systematic review and meta-analysis. Eur Heart J 34: 1807-1817, 2013.

KALAITZIDIS RG, ELISAF MS: The role of statins in chronic kidney disease. Am J Nephrol 34: 195-202, 2011.

KOIKE T, KITAJIMA S, YU Y, NISHIJIMA K, ZHANG J, OZAKI Y, MORIMOTO M, WATANABE T, BHAKDI S, ASADA Y, CHEN YE, FAN J: Human C-reactive protein does not promote atherosclerosis in transgenic rabbits. Circulation 120: 2088-2094, 2009.

KOPP JB: Rethinking hypertensive kidney disease: arterionephrosclerosis as a genetic, metabolic, and inflammatory disorder. Curr Opin Nephrol Hypertens 22: 266-272, 2013.

LI ZI, CHUNG AC, ZHOU L, HUANG XR, LIU F, FU P, FAN JM, SZALAI AJ, LAN HY: C-reactive protein promotes acute renal inflammation and fibrosis in unilateral ureteral obstructive nephropathy in mice. $L a b$ Invest 91: 837-851, 2011.

LIU F, CHEN HY, HUANG XR, CHUNG AC, ZHOU L, FU P, SZALAI AJ, LAN HY: C-reactive protein promotes diabetic kidney disease in a mouse model of type 1 diabetes. Diabetologia 54: 2713-2723, 2011.

MALÍNSKÁ H, OLIYARNYK O, HUBOVÁ M, ZÍDEK V, LANDA V, ŠIMÁKOVÁ M, MLEJNEK P, KAZDOVÁ L, KURTZ TW, PRAVENEC M: Increased liver oxidative stress and altered PUFA metabolism precede development of non-alcoholic steatohepatitis in SREBP-1a transgenic spontaneously hypertensive rats with genetic predisposition to hepatic steatosis. Mol Cell Biochem 335: 119-125, 2010.

MATHER KJ: The vascular endothelium in diabetes - a therapeutic target? Rev Endocr Metab Disord 14: 87-99, 2013.

NIKOLIC D, BANACH M, NIKFAR S, SALARI P, MIKHAILIDIS DP, TOTH PP, ABDOLLAHI M, RAY KK, PENCINA MJ, MALYSZKO J, RYSZ J, RIZZO M, LIPID AND BLOOD PRESSURE META-ANALYSIS COLLABORATION GROUP: A meta-analysis of the role of statins on renal outcomes in patients with chronic kidney disease. Is the duration of therapy important? Int J Cardio 168: 5437-5447, 2013.

OLSON JL: Hypertension: essential and secondary forms. In: Heptinstall's Pathology of the Kidney. 5th edn. JENNETTE JC, OLSON JL, SCHWARTZ M, SILVA FG (eds), Lippincott-Raven, Philadelphia, 1998, pp 943-1001.

PALMER SC, NAVANEETHAN SD, CRAIG JC, JOHNSON DW, PERKOVIC V, HEGBRANT J, STRIPPOLI GF: HMG CoA reductase inhibitors (statins) for people with chronic kidney disease not requiring dialysis. Cochrane Database Syst Rev 5: CD007784, 2014.

PRAVENEC M, KAJIYA T, ZÍDEK V, LANDA V, MLEJNEK P, ŠIMÁKOVÁ M, ŠILHAVÝ J, MALÍNSKÁ H, OLIYARNYK O, KAZDOVÁ L, FAN J, WANG J, KURTZ TW: Effects of human C-reactive protein on pathogenesis of features of the metabolic syndrome. Hypertension 57: 731-737, 2011. 
QASEEM A, HOPKINS RH, SWEET DE, STARKEY M, SHEKELLE P: Screening, monitoring, and treatment of stage 1 to 3 chronic kidney disease: a clinical practice guideline from the clinical guidelines committee of the American College of Physicians. Ann Intern Med 159: 835-847, 2013.

RIETZSCHEL E, DE BUYZERE M: High-sensitive C-reactive protein: universal prognostic and causative biomarker in heart disease? Biomark Med 6: 19-34, 2012.

SCIRICA BM, MORROW DA: Is C-reactive protein an innocent bystander or proatherogenic culprit? The verdict is still out. Circulation 113: 2128-2134, 2006.

ŠILHAVÝ J, ZÍDEK V, LANDA V, ŠIMÁKOVÁ M, MLEJNEK P, ŠKOP V, OLIYARNYK O, KAZDOVÁ L, MANCINI M, SAAR K, SCHULZ H, HÜBNER N, KURTZ TW, PRAVENEC M: Rosuvastatin can block pro-inflammatory actions of transgenic human CRP without reducing its circulating levels. Cardiovasc Ther 32: 59-65, 2014.

TERVAERT TW, MOOYAART AL, AMANN K, COHEN AH, COOK HT, DRACHENBERG CB, FERRARIO F, FOGO AB, HAAS M, DE HEER E, JOH K, NOËL LH, RADHAKRISHNAN J, SESHAN SV, BAJEMA IM, BRUIJN JA, RENAL PATHOLOGY SOCIETY: Pathologic classification of diabetic nephropathy. $J$ Am Soc Nephrol 21: 556-563, 2010.

VALK EJ, BRUIJN JA, BAJEMA IM: Diabetic nephropathy in humans: pathologic diversity. Curr Opin Nephrol Hypertens 20: 285-289, 2011.

WOLF G: New insights into the pathophysiology of diabetic nephropathy: from haemodynamics to molecular pathology. Eur J Clin Invest 34: 785-796, 2004.

YAGI S, AIHARA K, IKEDA Y, AKAIKE M, SATA M, MATSUMOTA T: Effects of statins on cardiorenal syndrome. Int J Vasc Med 2012: 162545, 2012.

ZHANG W, HE J, ZHANG F, HUANG C, WU Y, HAN Y, ZHANG W, ZHAO Y: Prognostic role of C-reactive protein and interleukin-6 in dialysis patients: a systematic review and meta-analysis. $J$ Nephrol 26: 243-253, 2013. 